\title{
sciendo
}

\section{THE PROTECTIVE ROLE OF VITAMINS (E + C) ON NILE TILAPIA (OREOCHROMIS NILOTICUS) EXPOSED TO ZNO NPS AND ZN IONS: BIOACCUMULATION AND PROXIMATE CHEMICAL COMPOSITION}

\author{
Amal Said Mohamed ${ }^{1,3 *}$, Hala Elshahat Ghannam ${ }^{1}$,Adel A. El-Lahamy ${ }^{2}$,Hanan Abdelhameid Soliman ${ }^{3}$ \\ 'Fresh Water Division, National Institute of Oceanography and Fisheries (NIOF), Egypt \\ ${ }^{2}$ Fisheries Division, National Institute of Oceanography and Fisheries (NIOF), Egypt \\ ${ }^{3}$ Faculty of Science, Biochemistry Division, Beni Suef University, Egypt \\ •Corresponding author: adelaml@yahoo.com \\ \#ORCID ID: https://orcid.org/0000-0001-9766-2884
}

\begin{abstract}
The accumulation potency of zinc nanoparticles in Nile tilapia (Oreochromis niloticus) was previously studied but their impacts on proximate chemical composition in muscle tissue by describing the dose-dependent accumulation and the protective role of vitamins ( $\mathrm{E}+\mathrm{C}$ ), have not been investigated. Therefore, this study was carried out to assess the protective role of vitamins $(\mathrm{E}+\mathrm{C})$ on $\mathrm{Zn}$ accumulation in muscle and gill tissues of $O$. niloticus exposed to three sub-lethal concentrations $\left(1 / 8 \mathrm{LC}_{50}, 1 / 4 \mathrm{LC}_{50}\right.$, and $\left.1 / 2 \mathrm{LC}_{50}\right)$ of zinc oxide nanoparticles (ZnO NPs) compared to zinc oxide bulk particles (ZnO BPs) as well as their effects on the induced chemical composition alterations for different experimental periods $(7,14,21$, and 28 days). The data displayed that fish exposed to the different sub-lethal concentrations of $\mathrm{ZnO}$ NPs or $\mathrm{ZnO}$ BPs have a significant increase $(\mathrm{P}<0.05)$ in $\mathrm{Zn}$ ions accumulation in muscle and gill tissues compared to control group but $\mathrm{Zn}$ was accumulated in gill tissue higher than muscle tissue at all exposure periods. Also, Zn accumulation was higher in fish tissues exposed to ZnO NPs than ZnO BPs. On the other hand, groups supplemented with vitamins (E + C) showed a significant decreasing $(\mathrm{P}<\mathbf{0 . 0 5})$ in accumulated $\mathrm{Zn}$ levels compared to groups without supplementation. The values of these supplemented groups returned to similar levels established in the control at low concentrations but still higher than control at the high concentrations. Furthermore, the results showed that moisture and ash content slightly increased while protein and fat decreased in fish exposed to $\mathrm{ZnO} \mathrm{NPs}$ or $\mathrm{ZnO} \mathrm{BPs}$ compared to control group. In conclusion, the findings supported that a combination of vitamins $(\mathrm{E}+\mathrm{C}) \mathrm{reduced} \mathrm{Zn}$ accumulation and ameliorated chemical composition alterations in $\boldsymbol{O}$. niloticus fish.
\end{abstract}

Key words: accumulation, $O$. niloticus, chemical composition, ZnO NPs, vitamins $(\mathrm{E}+\mathrm{C})$

The life standards of human societies have been rigorously changed after introduction of modern technologies. One of the most necessary technologies of the last century is definitely nanotechnology. The aquatic bodies are especially at risk of nanoparticles exposure; however, there are currently some reports revealing their safety in aquaculture (Neamat-Allah et al., 2019; Mahboub et al., 2021; Ibrahim et al., 2021) and scarce data about their bioaccumulation pattern in aquatic biota, their toxicological potency and their performance in aquatic habitats (Rundle et al., 2016; Khoei, 2021). Any particle with at least one dimension less than $100 \mathrm{~nm}$ is defined as nanoparticle, and consequently, the properties of these nanoparticles are altered compared to their bulk counterparts (Auffan et al., 2009). The nanoparticles can persist in the aquatic system for long period because of their slow degradation rate, and this would elevate their accumulation in the environment, magnification in the food chain, and long-term impacts on the ecosystems (García-Alonso et al., 2014).

Zinc oxide nanoparticles, one of the most widely used nanoparticles, are already produced in industrial scale (Peralta-Videa et al., 2011). In addition, they will certainly be used more widely in various industries due to their unique benefits in medicinal and industrial products. The exposure of ecosystems and humans to nanoparticles will also increase because of direct and indirect releases of nanoparticles into aquatic environments by sewage effluent and engineering applications (Collins et al., 2012). Recent studies demonstrated the immunotoxicity induced by ZnO NPs (Rashidian et al., 2021), while a safety side for the dietary inclusion of $\mathrm{ZnO}$ NPs was addressed in O. niloticus (Mahboub et al., 2020; Ghazi et al., 2021). Although some disagreements were found, the generally accepted toxicity mechanisms of $\mathrm{ZnO}$ NPs include particle and dissolved free ion impacts (Ma et al., 2013). Nile tilapia; O. niloticus become a necessary commercial fish in the world (El-Sayed, 2006). It could be used as a bio-indicator organism for indication of the effect of trace metals. This is because of its easy aquaculture, handling, and maintenance in the laboratory, also $O$. niloticus was used as a model organism for toxicological study because this species responds promptly to various environmental changes (Almeida et al., 2002; Garcia-Santos et al., 2006). Determination of proximate chemical composition is important to illustrate that fish tissues have healthy safe qualities and meet the international and national standard specifications (WHO/FAO, 2011). 
Vitamins are organic compounds important for health and normal growth; often are not synthesized via fish, and must be provided in the diet (El-Shebly, 2009). Vitamins $(\mathrm{C}, \mathrm{E}$, and $\mathrm{A})$ are the most significant antioxidant vitamins; where the vitamins $\mathrm{A}$ and $\mathrm{E}$ are considered as the primary lipid-soluble antioxidants that cooperate with vitamin $\mathrm{C}$ to prevent lipids from peroxidative damage (Choi et al., 2004; Mekkawy et al., 2012). Recent studies have assessed the toxicity of ZnO NPs and Zn ions on different aquatic organisms and reported oxidative stress, bioaccumulation, immunological, histopathological and ultrastructural changes (Kaya et al., 2015, 2016; Mansouri et al., 2018; Sayadi et al., 2020). Antioxidative vitamins such as $\mathrm{E}$ and $\mathrm{C}$, which are found in many food products, have been shown to have a protective role against nanoparticles and metals toxicity (Asaikkutti et al., 2016; Sahiti et al., 2018; Abdelazim et al., 2018; Mohamed et al., 2021) but there is no information available on the effect of these vitamins against $\mathrm{Zn}$ accumulation and proximate chemical composition alterations. Therefore, this study aims to determine the protective role of vitamins $(\mathrm{E}+\mathrm{C})$ in $O$. niloticus exposed to $\mathrm{ZnO}$ NPs compared to $\mathrm{ZnO}$ BPs and their impacts on $\mathrm{Zn}$ accumulation and proximate chemical composition alterations at different exposure periods.

\section{Material and methods}

\section{Materials preparation and fish acclimatization}

Zinc oxide bulk particles were purchased from ElNasr Pharmaceutical Chemicals Co. Egypt, while zinc oxide nanoparticles were obtained from Faculty of Postgraduate Studies for Advanced Sciences, Beni Suef University, Egypt, as detailed in our previous study (Mohamed et al., 2021), the same preparation and characterization (Figure 1). The different suspension concentrations (1/2, $1 / 4$, and $1 / 896 \mathrm{~h} \mathrm{LC}_{50}$ ) of both zinc oxide (bulk and nanoparticles) were freshly obtained by weighing dry $\mathrm{ZnO}$ powder in dechlorinated water $(\mathrm{pH}=7.4)$, then exposed to ultra sonication $(40 \mathrm{kHz}, 100 \mathrm{~W})$ for $\mathrm{h}$ to increase their dispersal.

O. niloticus were obtained from fish farm located at Fayoum governorate, The weight was $110 \pm 5 \mathrm{~g}$ and body length was $20 \pm 4 \mathrm{~cm}$. Fish were acclimated to laboratory conditions in fiberglass tanks (each of 1500-L capacity) containing dechlorinated tap water $(500-\mathrm{L}$ in each tank) for 2 weeks, a period that was enough to indicate the health status of the fish or appearance of any infectious diseases, before the start of the experiment. Fish were randomly distributed at a rate of 10 fish per tank and water quality parameters in the experimental tanks were analyzed according to the methods of American Public Health Association (APHA, 2005) (Table 1). Also, photoperiod was $12 \mathrm{~h}$ light: $12 \mathrm{~h}$ dark. Fish were fed (4\% of fish body weight) twice daily at 9:00 am and 4:00 pm with commercial pellet food ( $30 \%$ protein) which was used as control diet and the second diet was formulated as control diet plus vitamins $(E+C)(250$ $\mathrm{mg}$ of vitamin $\mathrm{E}+250 \mathrm{mg}$ of vitamin $\mathrm{C}$ ) given as mixtures per $\mathrm{kg}$ diet. Water was changed with routine clearing of the tanks, leaving no fecal matter and unconsumed food. Also, any fish showing unusual performances were excluded.

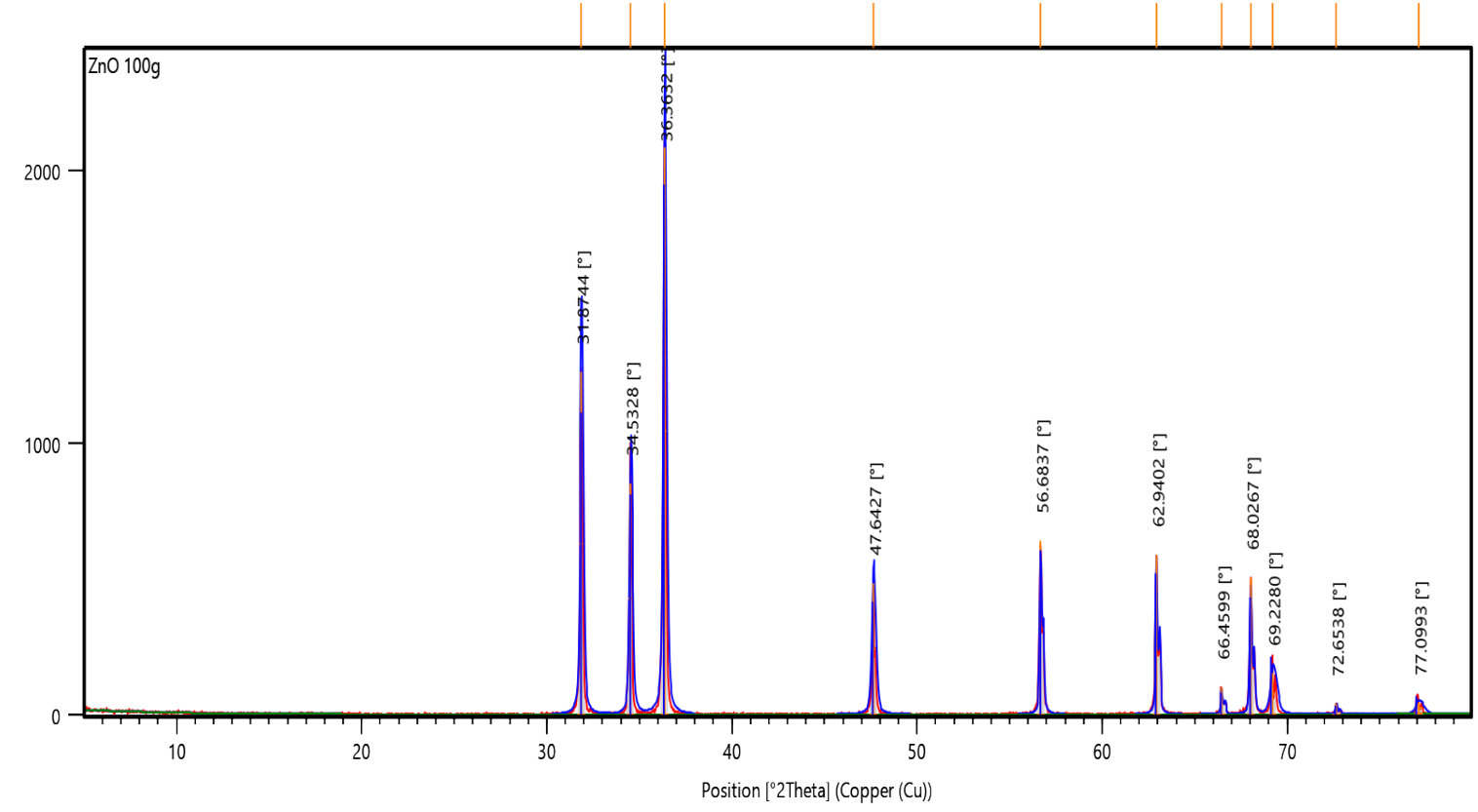

Figure 1. X-ray diffraction (XRD) pattern of ZnONPs as described in Mohamed et al. (2021) 
Table 1. Range of water quality parameters during the acclimatization and experimental period

\begin{tabular}{l|l}
\hline \multicolumn{1}{c|}{ Parameter } & \multicolumn{1}{c}{ Range } \\
\hline Temp & $27.1-27.3^{\circ} \mathrm{C}$ \\
$\mathrm{pH}$ & $7.38-7.51$ \\
$\mathrm{EC}$ & $637-645 \mu \mathrm{S} / \mathrm{cm}$ \\
DO & $6.8-7.2 \mathrm{mg} / \mathrm{l}$ \\
BOD & $2.4-3.4 \mathrm{mg} / \mathrm{l}$ \\
COD & $4.8-5.6 \mathrm{mg} / \mathrm{l}$ \\
ammonia & $0.120-0.170 \mathrm{mg} / 1$ \\
nitrite & $9.08-9.99 \mu \mathrm{g} / \mathrm{l}$ \\
nitrate & $33.7-34.1 \mu \mathrm{g} / 1$ \\
\hline
\end{tabular}

Temp: Temperature, $\mathrm{pH}$ : Hydrogen ion concentration, EC: electric conductivity, DO: Dissolved oxygen, BOD: Biological oxygen demand, COD: Chemical oxygen demand.

\section{Experimental design}

After two weeks of acclimatization, a total number of 390 males $O$. niloticus fish were divided into 13 experimental groups in a triplicate manner. Each group consisted of 30 fish triplicate (10 fish/aquarium). Fish of the different studied groups were exposed to three sub-lethal concentrations $\left(1 / 8 \mathrm{LC}_{50}, 1 / 4 \mathrm{LC}_{50}\right.$ and $\left.1 / 2 \mathrm{LC}_{50}\right)$ of $\mathrm{ZnO}$ BPs and $\mathrm{ZnO}$ NPs, i.e. 10.5, 21 and $42 \mathrm{mg} / \mathrm{L}$ and 0.7, 1.4 and $2.8 \mathrm{mg} / \mathrm{L}$, respectively based on Mohamed et al. (2021); $96 \mathrm{~h} \mathrm{LC}_{50}$ value of $\mathrm{ZnO} \mathrm{BPs}$ and $\mathrm{ZnO}$ NPs for $O$. niloticus fish was $84 \mathrm{mg} / \mathrm{l}$ and $5.6 \mathrm{mg} / \mathrm{l}$ respectively. Also, fish were exposed to the same sub-lethal concentrations of $\mathrm{ZnO}$ BPs or $\mathrm{ZnO}$ NPs plus vitamins $(\mathrm{E}+\mathrm{C})(500$ $\mathrm{mg} / \mathrm{kg}$ diet) as follows:

G1: The first group served as a control, G2, G3, and G4: The three experimental groups exposed to three sublethal concentrations $\left(1 / 8 \mathrm{LC}_{50}, 1 / 4 \mathrm{LC}_{50}\right.$ and $\left.1 / 2 \mathrm{LC}_{50}\right)$ of $\mathrm{ZnO}$ BPs respectively. G5, G6, and G7: Groups of fish exposed to $1 / 8 \mathrm{LC}_{50}, 1 / 4 \mathrm{LC}_{50}$, and $1 / 2 \mathrm{LC}_{50}$ of $\mathrm{ZnO}$ BPs respectively and supplemented with vitamins $(\mathrm{E}+$ C) $(500 \mathrm{mg} / \mathrm{kg}$ diet $)$. G8, G9, and G10: Fish exposed to three sub-lethal concentrations $\left(1 / 8 \mathrm{LC}_{50}, 1 / 4 \mathrm{LC}_{50}\right.$ and $1 / 2 \mathrm{LC}_{50}$ ) of ZnO NPs respectively. G11, G12, and G13: Fish exposed to $1 / 8 \mathrm{LC}_{50}, 1 / 4 \mathrm{LC}_{50}$ and $1 / 2 \mathrm{LC}_{50}$ of $\mathrm{ZnO}$ NPs respectively plus vitamins $(\mathrm{E}+\mathrm{C})(500 \mathrm{mg} / \mathrm{kg}$ diet $)$.

The experimental periods were $7,14,21$, and 28 days for all groups. The conditions of the experiments were as those of the acclimatization period, and water was daily checked for temperature, dissolved oxygen and $\mathrm{pH}$. After 7, 14, 21 and 28 days of exposure periods, fish samples were taken from different groups for assessment of zinc accumulation and proximate chemical composition.

\section{Determination of $\mathrm{Zn}$ concentrations in fish tissue}

For determining $\mathrm{Zn}$ residues in the investigated fish organs (muscle and gill), about $5 \mathrm{~g}$ of fresh fish tissue specimens were dried in an oven at $105^{\circ} \mathrm{C}$ for 2 days and ground to a fine powder. Samples were digested after drying according to the method of Ghazally (1988) in which dry powder $(1.0 \mathrm{~g})$ was digested in a solution of 5 $\mathrm{ml}$ perchloric acid $+5 \mathrm{ml}$ nitric acid, boiled at $80-90^{\circ} \mathrm{C}$ on hot plate until the sample became clear. The solution was filtrated after cooling and transferred to $25 \mathrm{ml}$ volumetric flask then filled up to the level with de-ionized water. The samples were kept in plastic bottles and later the levels of $\mathrm{Zn}$ in gill and muscle were measured by GBC atomic absorption spectrophotometer Savanta AA.

\section{Proximate chemical composition analyses}

Muscles of all experimental fishes were immediately removed and taken (fresh muscles tissue). The proximate chemical composition analyses (moisture, crude protein, crude fat, and ash content) in fish muscle were determined according to AOAC (2012). Moisture content was determined by drying the muscle samples in a heated oven (GCA, model 18EM, Precision Scientific Group, Chicago, Illinois, USA) at $85^{\circ} \mathrm{C}$ for 2 days. Nitrogen content was measured by a micro-Kjeldahl apparatus (Labconco, Labconco Corporation, Kansas, Missouri, USA) and crude protein by multiplying nitrogen content by 6.25 . Fat content was measured by ether extraction in multi-unit extraction Soxhlet apparatus (Lab-Line Instruments, Inc., Melrose Park, Illinois, USA) for 16 h. Also, ash content was evaluated by combusting dry samples in a muffle furnace (Thermolyne Corporation, Dubuque, Iowa, USA) at $550^{\circ} \mathrm{C}$ for $6 \mathrm{~h}$. Finally, total carbohydrates were estimated using the following equation (Merrill and Watt, 1973): Total carbohydrates $=100 \%-(\%$ fat $+\%$ protein $+\%$ moisture $+\%$ ash).

\section{Statistical analysis}

Data were statistically analyzed using analysis of variance (one-way ANOVA) and significant differences between groups were determined by Duncan Waller Multiple Range Test using SPSS statistical package version 17. All results were expressed as mean \pm standard error $(\mathrm{M} \pm \mathrm{SE})$. The significance level (P value) was used to answer if the difference significant or not, where: $(\mathrm{P}<0.05)$ $=$ significant and $\mathrm{P}>0.05=$ not significant. We used $\mathrm{R}$ ( $\mathrm{R}$ Core Team, 2017) for generating all figures. These figures were produced using the ggplot 2 package (Wickham, 2016).

\section{Results}

\section{Zinc (Zn) accumulation in fish tissues}

The changes in the concentration of $\mathrm{Zn}$ accumulated in muscle and gill tissues of $O$. niloticus exposed to sub-lethal concentrations of $\mathrm{ZnO} \mathrm{BPs}$ or $\mathrm{ZnO}$ NPs and supplemented with vitamins $(E+C)$ after the exposure periods $(7,14,21$, and 28 days) were represented in Tables $(2-3)$.

\section{Concentration of $\mathrm{Zn}$ in muscle tissue}

The results indicated that the concentration of $\mathrm{Zn}$ accumulated in muscle tissue of $O$. niloticus exposed to sub-lethal concentrations of $\mathrm{ZnO}$ BPs or ZnO NPs was increased significantly $(\mathrm{P} \leq 0.05)$ compared to the control 
group after $7,14,21$, and 28 days except $1 / 8 \mathrm{LC}_{50}$ of $\mathrm{ZnO}$ NPs after 7 days. The group exposed to the higher concentrations (1/2 LC50) have accumulated higher level of $\mathrm{Zn}$ in muscles than the group exposed to the lower concentrations $\left(1 / 8 \mathrm{LC}_{50}\right)$. Also, fish groups exposed to $\mathrm{ZnO}$ NPs accumulated $\mathrm{Zn}$ higher than that of $\mathrm{ZnO}$ BPs at all exposure periods. However, fish group exposed to the same concentration of ZnO BPs or ZnO NPs and supplemented with vitamins ( $\mathrm{E}$ and $\mathrm{C}$ ) showed a significant decreasing $(\mathrm{P} \leq 0.05)$ in accumulated $\mathrm{Zn}$ level compared to the group without supplementation after all exposure periods except $1 / 8 \mathrm{LC}_{50}$ of $\mathrm{ZnO}$ NPs plus vitamins (E $+\mathrm{C})$ at 7 days. Also, the levels of groups supplemented with vitamins returned to similar levels established in the control at low concentrations but still higher than control at the higher concentrations (Table 2).

\section{Concentration of $\mathrm{Zn}$ in gill tissue}

A significant increase $(\mathrm{P} \leq 0.05)$ was found in the concentration of $\mathrm{Zn}$ accumulated in gill tissue of $O$. niloti- cus exposed to sub-lethal concentrations of $\mathrm{ZnO}$ BPs or $\mathrm{ZnO}$ NPs compared to the control group after 7, 14, 21, and 28 days. Accumulation of $\mathrm{Zn}$ showed a significant increase $(\mathrm{P} \leq 0.05)$ by increasing $\mathrm{ZnO}$ BPs or $\mathrm{ZnO}$ NPs concentrations and the group exposed to the higher concentrations $\left(1 / 2 \mathrm{LC}_{50}\right)$ have accumulated higher level of $\mathrm{Zn}$ in gill than the group exposed to the lower concentrations $\left(1 / 8 \mathrm{LC}_{50}\right)$. In addition, fish groups exposed to $\mathrm{ZnO}$ NPs accumulated $\mathrm{Zn}$ higher than that of $\mathrm{ZnO}$ BPs at all exposure periods. On the other hand, fish group exposed to the same concentration of $\mathrm{ZnO}$ BPs or $\mathrm{ZnO}$ NPs and supplemented with vitamins $(E+C)$ showed a significant decrease $(\mathrm{P} \leq 0.05)$ in accumulated $\mathrm{Zn}$ level compared to the group without supplementation after all studied periods as well as the values of these supplemented groups returned to similar levels established in the control at low concentrations but still higher than control at the higher concentrations. Also, gill tissue accumulated higher level of $\mathrm{Zn}$ than muscle tissue of $O$. niloticus after all studied exposure periods (Table 3 ).

Table 2. Zn accumulation (mg/kg dry weight) in muscle tissue of $O$. niloticus exposed to ZnO BPs or ZnO NPs sub-lethal concentrations and vitamins $(\mathrm{E}+\mathrm{C})$ supplementation

\begin{tabular}{|c|c|c|c|c|c|c|c|}
\hline \multirow{2}{*}{$\begin{array}{l}\text { Time } \\
\text { (days) }\end{array}$} & \multirow[t]{2}{*}{ Control } & \multicolumn{3}{|c|}{ Sub-lethal concentration } & \multicolumn{3}{|c|}{$\begin{array}{l}\text { Sub-lethal concentration }+ \text { vitamins } \\
\qquad(\mathrm{E}+\mathrm{C})\end{array}$} \\
\hline & & $1 / 8 \mathrm{LC}_{50}$ & $1 / 4 \mathrm{LC}_{50}$ & $1 / 2 \mathrm{LC}_{50}$ & $1 / 8 \mathrm{LC}_{50}$ & $1 / 4 \mathrm{LC}_{50}$ & $1 / 2 \mathrm{LC}_{50}$ \\
\hline \multicolumn{8}{|l|}{$\mathrm{ZnOBPs}$} \\
\hline 7 & $20.3 \pm 0.17 \mathrm{~d}$ & $21.6 \pm 0.34 \mathrm{bc}$ & $22 \pm 0.57 \mathrm{ab}$ & $23.2 \pm 0.05 \mathrm{a}$ & $20.4 \pm 0.23 \mathrm{~d}$ & $20.7 \pm 0.40 \mathrm{~cd}$ & $20.8 \pm 0.46 \mathrm{bcd}$ \\
\hline 14 & $20.9 \pm 0.51 \mathrm{~d}$ & $22.2 \pm 0.11 \mathrm{c}$ & $23.6 \pm 0.34 \mathrm{~b}$ & $25.3 \pm 0.17 \mathrm{a}$ & $21 \pm 0.54 \mathrm{~cd}$ & $21.6 \pm 0.34 \mathrm{~cd}$ & $21.7 \pm 0.28 \mathrm{~cd}$ \\
\hline 21 & $21 . .2 \pm 0.69 \mathrm{c}$ & $25.4 \pm 0.40 \mathrm{~b}$ & $28.9 \pm 0.51 \mathrm{a}$ & $31 \pm 1.15 \mathrm{a}$ & $23.4 \pm 0.80 \mathrm{bc}$ & $23.7 \pm 0.98 \mathrm{bc}$ & $24.9 \pm 0.51 \mathrm{~b}$ \\
\hline 28 & $21.6 \pm 0.23 \mathrm{e}$ & $28.1 \pm 0.57 \mathrm{~cd}$ & $30.7 \pm 0.40 \mathrm{~b}$ & $39.7 \pm 0.98 \mathrm{a}$ & $26.9 \pm 0.51 \mathrm{~d}$ & $28.1 \pm 063 \mathrm{~cd}$ & $29.4 \pm 0.23 \mathrm{bc}$ \\
\hline \multicolumn{8}{|l|}{ ZnONPs } \\
\hline 7 & $20.3 \pm 0.17 \mathrm{~b}$ & $21.9 \pm 0.14 \mathrm{~b}$ & $23.8 \pm 1.03 \mathrm{a}$ & $24.1 \pm 0.57 \mathrm{a}$ & $21 \pm 0.28 \mathrm{~b}$ & $21.1 \pm 0.05 \mathrm{~b}$ & $21.6 \pm 0.17 \mathrm{~b}$ \\
\hline 14 & $20.9 \pm 0.51 \mathrm{~d}$ & $25.3 \pm 0.17 \mathrm{~b}$ & $25.7 \pm 0.69 \mathrm{~b}$ & $27.5 \pm 0.86 \mathrm{a}$ & $21.6 \pm 0.34 \mathrm{~cd}$ & $22.7 \pm 0.11 \mathrm{c}$ & $22.8 \pm 0.23 \mathrm{c}$ \\
\hline 21 & $21 . .2 \pm 0.69 \mathrm{e}$ & $28.4 \pm 0.08 \mathrm{bc}$ & $29.1 \pm 0.63 \mathrm{~b}$ & $31.9 \pm 0.51 \mathrm{a}$ & $23.6 \pm 0.92 \mathrm{~d}$ & $23.9 \pm 0.25 \mathrm{~d}$ & $26.8 \pm 0.28 \mathrm{c}$ \\
\hline 28 & $21.6 \pm 0.23 \mathbf{e}$ & $32.7 \pm 0.31 \mathbf{c}$ & $35.4 \pm 0.23 \mathbf{b}$ & $42.7 \pm 1.15 \mathbf{a}$ & $28.9 \pm 0.51 \mathbf{d}$ & $29.7 \pm 0.34 \mathbf{d}$ & $29.8 \pm 0.46 \mathbf{d}$ \\
\hline
\end{tabular}

Data are presented as mean $\pm \mathrm{SE}$ of 6 fish. SE: standard error.

$(\mathrm{a}, \mathrm{b}, \mathrm{c} \ldots$...) means within the same row carrying different letters are significant at $\mathrm{P} \leq 0.05$.

Means having the same letter in the same row are not significantly different.

Table 3. $\mathrm{Zn}$ accumulation (mg/kg dry weight) in gill tissue of O. niloticus exposed to $\mathrm{ZnO}$ BPs or ZnO NPs sub-lethal concentrations and vitamins $(\mathrm{E}+\mathrm{C})$ supplementation

\begin{tabular}{|c|c|c|c|c|c|c|c|}
\hline \multirow{2}{*}{$\begin{array}{l}\text { Time } \\
\text { (days) }\end{array}$} & \multirow[t]{2}{*}{ Control } & \multicolumn{3}{|c|}{ Sub-lethal concentration } & \multicolumn{3}{|c|}{$\begin{array}{l}\text { Sub-lethal concentration }+ \text { vitamins } \\
\qquad(E+C)\end{array}$} \\
\hline & & $1 / 8 \mathrm{LC}_{50}$ & $1 / 4 \mathrm{LC}_{50}$ & $1 / 2 \mathrm{LC}_{50}$ & $1 / 8 \mathrm{LC}_{50}$ & $1 / 4 \mathrm{LC}_{50}$ & $1 / 2 \mathrm{LC}_{50}$ \\
\hline \multicolumn{8}{|l|}{$\mathrm{ZnOBPs}$} \\
\hline 7 & $60.0 \pm 0.57 \mathrm{~d}$ & $68 \pm 1.15 \mathrm{c}$ & $76 \pm 2.30 \mathrm{~b}$ & $87 \pm 1.73 \mathrm{a}$ & $61 \pm 1.15 \mathrm{~d}$ & $61.5 \pm 0.86 \mathrm{~d}$ & $63 \pm 2.30 \mathrm{~cd}$ \\
\hline 14 & $60.5 \pm 1.15 \mathrm{~d}$ & $74 \pm 2.30 \mathrm{c}$ & $88 \pm 4.61 \mathrm{~b}$ & $114 \pm 8.08 \mathrm{a}$ & $62.5 \pm 1.44 \mathrm{~cd}$ & $64 \pm 1$ & $69 \pm 1.73 \mathrm{~d}$ \\
\hline 21 & $60.5 \pm 1.73 \mathrm{e}$ & $103 \pm 4.61 \mathrm{c}$ & $122 \pm 6.92 \mathrm{~b}$ & $146 \pm 9.23 \mathrm{a}$ & $71 \pm 3.46 \mathrm{de}$ & $76 \pm 3.46 \mathrm{de}$ & $87 \pm 2.88 \mathrm{~cd}$ \\
\hline 28 & $61.5 \pm 2.30 \mathrm{e}$ & $120 \pm 3.46 \mathrm{c}$ & $190 \pm 4.61 \mathrm{~b}$ & $230 \pm 8.66 \mathrm{a}$ & $90 \pm 230 \mathrm{~d}$ & $101 \pm 4.04 \mathrm{de}$ & $108 \pm 1.73 \mathrm{~cd}$ \\
\hline \multicolumn{8}{|l|}{ ZnONPs } \\
\hline 7 & $60.0 \pm 0.57 \mathrm{e}$ & $72 \pm 2.30 \mathrm{c}$ & $89.0 \pm 1.73 \mathrm{~b}$ & $111 \pm 2.88 \mathrm{a}$ & $62 \pm 0.57 \mathrm{de}$ & $64 \pm 1.73 \mathrm{de}$ & $67 \pm 1.15 \mathrm{~cd}$ \\
\hline 14 & $60.5 \pm 1.15 \mathrm{f}$ & $80 \pm 5.19 \mathrm{c}$ & $102 \pm 4.04 \mathrm{~b}$ & $134 \pm 2.30 \mathrm{a}$ & $60 \pm 1.73$ ef & $70 \pm 1.44 \mathrm{de}$ & $78 \pm 0.86 \mathrm{~cd}$ \\
\hline 21 & $60.5 \pm 1.73 \mathrm{e}$ & $112 \pm 4.04 \mathrm{c}$ & $141.5 \pm 11.5 \mathrm{~b}$ & $181 \pm 6.35 \mathrm{a}$ & $75 \pm 2.88 \mathrm{de}$ & $86 \pm 3.46 \mathrm{~d}$ & $94 \pm 1.15 \mathrm{~cd}$ \\
\hline 28 & $61.5 \pm 2.30 \mathrm{e}$ & $140 \pm 2.88 \mathrm{~b}$ & $270 \pm 11.5 \mathrm{a}$ & $270 \pm 2.88 \mathrm{a}$ & $92 \pm 1.73 \mathrm{~d}$ & $113 \pm 2.30 \mathrm{c}$ & $120 \pm 3.46 \mathrm{c}$ \\
\hline
\end{tabular}

Data are presented as mean $\pm \mathrm{SE}$ of 6 fish. SE: standard error.

(a, b, c ...) means within the same row carrying different letters are significant at $\mathrm{P} \leq 0.05$.

Means having the same letter in the same row are not significantly different. 
Table 4. Moisture content (\%) of $O$. niloticus exposed to ZnO BPs or ZnO NPs sub-lethal concentrations and vitamins (E + C) supplementation

\begin{tabular}{|c|c|c|c|c|c|c|c|}
\hline \multirow{2}{*}{$\begin{array}{l}\text { Time } \\
\text { (days) }\end{array}$} & \multirow{2}{*}{ Control } & \multicolumn{3}{|c|}{ Sub-lethal concentration } & \multicolumn{3}{|c|}{$\begin{array}{l}\text { Sub-lethal concentration }+ \text { vitamins } \\
\qquad(E+C)\end{array}$} \\
\hline & & $1 / 8 \mathrm{LC}_{50}$ & $1 / 4 \mathrm{LC}_{50}$ & $1 / 2 \mathrm{LC}_{50}$ & $1 / 8 \mathrm{LC}_{50}$ & $1 / 4 \mathrm{LC}_{50}$ & $1 / 2 \mathrm{LC}_{50}$ \\
\hline \multicolumn{8}{|l|}{$\mathrm{ZnOBPs}$} \\
\hline 7 & $79.55 \pm 0.31$ & $79.87 \pm 0.21$ & $80.00 \pm 0.57$ & $80.36 \pm 0.17$ & $79.64 \pm 0.13$ & $79.84 \pm 0.25$ & $80.08 \pm 0.10$ \\
\hline 14 & $79.00 \pm 0.23$ & $79.33 \pm 0.19$ & $79.44 \pm 0.25$ & $79.82 \pm 0.47$ & $79.07 \pm 0.61$ & $79.22 \pm 0.70$ & $79.51 \pm 0.23$ \\
\hline 21 & $78.60 \pm 0.34$ & $78.82 \pm 0.28$ & $79.00 \pm 0.86$ & $79.23 \pm 0.13$ & $78.61 \pm 0.35$ & $78.85 \pm 0.20$ & $79.02 \pm 0.58$ \\
\hline 28 & $78.13 \pm 0.65$ & $78.48 \pm 0.57$ & $78.67 \pm 0.87$ & $78.96 \pm 0.55$ & $78.24 \pm 071$ & $78.48 \pm 0.27$ & $78.65 \pm 0.37$ \\
\hline \multicolumn{8}{|l|}{ ZnONPs } \\
\hline 7 & $79.55 \pm 0.31$ & $80.32 \pm 0.18$ & $80.90 \pm 0.51$ & $80.92 \pm 0.24$ & $79.90 \pm 0.57$ & $80.26 \pm 0.73$ & $80.37 \pm 0.21$ \\
\hline 14 & $79.00 \pm 0.23$ & $79.76 \pm 0.43$ & $80.36 \pm 0.20$ & $80.38 \pm 0.57$ & $79.34 \pm 0.34$ & $79.72 \pm 0.12$ & $79.81 \pm 0.46$ \\
\hline 21 & $78.60 \pm 0.34$ & $79.12 \pm 0.64$ & $79.74 \pm 0.42$ & $79.60 \pm 0.20$ & $78.80 \pm 0.34$ & $79.27 \pm 0.05$ & $79.33 \pm 0.19$ \\
\hline 28 & $78.13 \pm 0.65$ & $78.94 \pm 0.54$ & $79.53 \pm 0.30$ & $79.53 \pm 0.23$ & $78.53 \pm 0.88$ & $78.91 \pm 0.52$ & $78.97 \pm 0.56$ \\
\hline
\end{tabular}

Data are presented as mean $\pm \mathrm{SE}$ of 6 fish. SE: standard error.

Table 5. Protein content (\%) of O. niloticus exposed to ZnO BPs or ZnO NPs sub-lethal concentrations and vitamins (E + C) supplementation

\begin{tabular}{|c|c|c|c|c|c|c|c|}
\hline \multirow{2}{*}{$\begin{array}{l}\text { Time } \\
\text { (days) }\end{array}$} & \multirow{2}{*}{ Control } & \multicolumn{3}{|c|}{ Sub-lethal concentration } & \multicolumn{3}{|c|}{$\begin{array}{l}\text { Sub-lethal concentration }+ \text { vitamins } \\
\qquad(E+C)\end{array}$} \\
\hline & & $1 / 8 \mathrm{LC}_{50}$ & $1 / 4 \mathrm{LC}_{50}$ & $1 / 2 \mathrm{LC}_{50}$ & $1 / 8 \mathrm{LC}_{50}$ & $1 / 4 \mathrm{LC}_{50}$ & $1 / 2 \mathrm{LC}_{50}$ \\
\hline \multicolumn{8}{|l|}{$\mathrm{ZnOBPs}$} \\
\hline 7 & $17.16 \pm 0.09$ & $16.99 \pm 0.28$ & $16.81 \pm 0.17$ & $16.65 \pm 0.37$ & $17.04 \pm 0.11$ & $16.95 \pm 0.20$ & $16.79 \pm 0.10$ \\
\hline 14 & $17.64 \pm 0.38$ & $17.49 \pm 0.28$ & $17.31 \pm 0.17$ & $17.12 \pm 0.06$ & $17.55 \pm 0.31$ & $17.43 \pm 0.24$ & $17.26 \pm 015$ \\
\hline 21 & $17.99 \pm 0.57$ & $17.76 \pm 0.43$ & $17.63 \pm 0.36$ & $17.43 \pm 0.24$ & $17.91 \pm 052$ & $17.76 \pm 0.43$ & $17.59 \pm 0.34$ \\
\hline 28 & $18.08 \pm 0.57$ & $17.91 \pm 0.23$ & $17.75 \pm 0.43$ & $17.46 \pm 0.26$ & $17.98 \pm 0.27$ & $17.87 \pm 0.50$ & $17.65 \pm 0.37$ \\
\hline \multicolumn{8}{|l|}{ ZnONPs } \\
\hline 7 & $17.16 \pm 0.09$ & $16.75 \pm 0.43$ & $16.2 \pm 0.11$ & $16.25 \pm 0.14$ & $16.91 \pm 0.52$ & $16.59 \pm 0.34$ & $16.55 \pm 0.31$ \\
\hline 14 & $17.64 \pm 0.38 \mathrm{a}$ & $17.07 \pm 0.04 \mathrm{ab}$ & $16.48 \pm 0.27 \mathrm{~b}$ & $16.56 \pm 0.32 \mathrm{ab}$ & $17.22 \pm 0.12 \mathrm{ab}$ & $16.89 \pm 0.22 \mathrm{ab}$ & $16.83 \pm 0.47 \mathrm{ab}$ \\
\hline 21 & $17.99 \pm 0.57$ & $17.41 \pm 0.23$ & $16.82 \pm 0.18$ & $16.9 \pm 0.51$ & $17.58 \pm 0.33$ & $17.23 \pm 0.13$ & $17.16 \pm 009$ \\
\hline 28 & $18.08 \pm 0.57$ & $17.65 \pm 0.37$ & $17.3 \pm 0.17$ & $17.11 \pm 0.06$ & $17.82 \pm 0.47$ & $17.45 \pm 0.25$ & $17.33 \pm 019$ \\
\hline
\end{tabular}

Data are presented as mean \pm SE of 6 fish. SE: standard error.

(a, b, c ...) means within the same row carrying different letters are significant at $\mathrm{P} \leq 0.05$.

Means having the same letter in the same row are not significantly different.

Table 6. Fat content (\%) of O. niloticus exposed to ZnO BPs or ZnO NPs sub-lethal concentrations and vitamins (E + C) supplementation

\begin{tabular}{|c|c|c|c|c|c|c|c|}
\hline \multirow{2}{*}{$\begin{array}{l}\text { Time } \\
\text { (days) }\end{array}$} & \multirow{2}{*}{ Control } & \multicolumn{3}{|c|}{ Sub-lethal concentration } & \multicolumn{3}{|c|}{$\begin{array}{l}\text { Sub-lethal concentration }+ \text { vitamins } \\
\qquad(E+C)\end{array}$} \\
\hline & & $1 / 8 \mathrm{LC}_{50}$ & $1 / 4 \mathrm{LC}_{50}$ & $1 / 2 \mathrm{LC}_{50}$ & $1 / 8 \mathrm{LC}_{50}$ & $1 / 4 \mathrm{LC}_{50}$ & $1 / 2 \mathrm{LC}_{50}$ \\
\hline \multicolumn{8}{|l|}{$\mathrm{ZnOBPs}$} \\
\hline 7 & $1.35 \pm 0.02 \mathrm{a}$ & $1.28 \pm 0.04 \mathrm{abc}$ & $1.21 \pm 0.06 \mathrm{bcd}$ & $1.10 \pm 0.02 \mathrm{~d}$ & $1.32 \pm 0.04 \mathrm{ab}$ & $1.25 \pm 0.02 \mathrm{abc}$ & $1.15 \pm 0.03 \mathrm{~cd}$ \\
\hline 14 & $1.48 \pm 0.04$ & $1.43 \pm 0.07$ & $1.34 \pm 0.02$ & $1.25 \pm 0.02$ & $1.45 \pm 0.08$ & $1.37 \pm 0.09$ & $1.27 \pm 0.04$ \\
\hline 21 & $1.73 \pm 0.07 \mathrm{a}$ & $1.63 \pm 0.05 \mathrm{ab}$ & $1.58 \pm 0.04 \mathrm{ab}$ & $1.49 \pm 0.05 \mathrm{~b}$ & $1.71 \pm 0.06 \mathrm{ab}$ & $1.63 \pm 0.07 \mathrm{ab}$ & $1.51 \pm 0.05 \mathrm{ab}$ \\
\hline 28 & $1.95 \pm 0.08$ & $1.90 \pm 0.11$ & $1.71 \pm 0.06$ & $1.60 \pm 0.04$ & $1.90 \pm 0.02$ & $1.81 \pm 0.17$ & $1.65 \pm 0.011$ \\
\hline \multicolumn{8}{|l|}{$\mathrm{ZnONPs}$} \\
\hline 7 & $1.35 \pm 0.02 \mathrm{a}$ & $1.2 \pm 0.04 \mathrm{abc}$ & $1.09 \pm 0.05 \mathrm{bcd}$ & $1.00 \pm 0.02 \mathrm{~d}$ & $1.26 \pm 0.03 \mathrm{ab}$ & $1.18 \pm 0.10 \mathrm{abcd}$ & $1.05 \pm 0.04 \mathrm{~cd}$ \\
\hline 14 & $1.48 \pm 0.04 \mathrm{a}$ & $1.35 \pm 0.02 \mathrm{ab}$ & $1.22 \pm 0.01 \mathrm{bc}$ & $1.13 \pm 0.01 \mathrm{c}$ & $1.41 \pm 0.07 \mathrm{ab}$ & $1.31 \pm 0.05 \mathrm{abc}$ & $1.19 \pm 0.10 \mathrm{bc}$ \\
\hline 21 & $1.73 \pm 0.07 \mathrm{a}$ & $1.58 \pm 0.04 \mathrm{ab}$ & $1.46 \pm 0.03 \mathrm{ab}$ & $1.35 \pm 0.08 \mathrm{~b}$ & $1.68 \pm 0.10 \mathrm{a}$ & $1.53 \pm 0.13 \mathrm{ab}$ & $1.43 \pm 0.07 \mathrm{ab}$ \\
\hline 28 & $1.95 \pm 0.08 \mathrm{a}$ & $1.51 \pm 0.05 \mathrm{bcd}$ & $1.2 \pm 0.11 \mathrm{~d}$ & $1.34 \pm 0.08 \mathrm{~cd}$ & $1.79 \pm 0.05 \mathrm{ab}$ & $1.68 \pm 0.04 \mathrm{ab}$ & $1.55 \pm 0.14 \mathrm{bc}$ \\
\hline
\end{tabular}

Data are presented as mean \pm SE of 6 fish. SE: standard error.

(a, b, c ...) means within the same row carrying different letters are significant at $\mathrm{P} \leq 0.05$.

Means having the same letter in the same row are not significantly different. 
Table 7. Ash content (\%) of O. niloticus exposed to $\mathrm{ZnO}$ BPs or $\mathrm{ZnO}$ NPs sub-lethal concentrations and vitamins (E + C) supplementation

\begin{tabular}{|c|c|c|c|c|c|c|c|}
\hline \multirow{2}{*}{$\begin{array}{l}\text { Time } \\
\text { (days) }\end{array}$} & \multirow{2}{*}{ Control } & \multicolumn{3}{|c|}{ Sub-lethal concentration } & \multicolumn{3}{|c|}{$\begin{array}{l}\text { Sub-lethal concentration }+ \text { vitamins } \\
\qquad(E+C)\end{array}$} \\
\hline & & $1 / 8 \mathrm{LC}_{50}$ & $1 / 4 \mathrm{LC}_{50}$ & $1 / 2 \mathrm{LC}_{50}$ & $1 / 8 \mathrm{LC}_{50}$ & $1 / 4 \mathrm{LC}_{50}$ & $1 / 2 \mathrm{LC}_{50}$ \\
\hline \multicolumn{8}{|l|}{$\mathrm{ZnOBPs}$} \\
\hline 7 & $1.26 \pm 0.02 \mathrm{~b}$ & $1.35 \pm 0.03 \mathrm{ab}$ & $1.39 \pm 0.05 \mathrm{ab}$ & $1.49 \pm 0.04 \mathrm{a}$ & $1.31 \pm 0.05 \mathrm{ab}$ & $1.36 \pm 0.02 \mathrm{ab}$ & $1.45 \pm 0.08 \mathrm{a}$ \\
\hline 14 & $1.32 \pm 0.03 \mathrm{~b}$ & $1.44 \pm 0.02 \mathrm{ab}$ & $1.48 \pm 0.04 \mathrm{ab}$ & $1.50 \pm 0.05 \mathrm{a}$ & $1.40 \pm 0.05 \mathrm{ab}$ & $1.45 \pm 0.02 \mathrm{ab}$ & $1.56 \pm 0.06 \mathrm{a}$ \\
\hline 21 & $1.42 \pm 0.01 \mathrm{~b}$ & $1.51 \pm 0.06 \mathrm{abc}$ & $1.60 \pm 0.02 \mathrm{ab}$ & $1.63 \pm 0.02 \mathrm{ab}$ & $1.49 \pm 0.05 \mathrm{bc}$ & $1.58 \pm 0.04 \mathrm{abc}$ & $1.68 \pm 0.08 \mathrm{a}$ \\
\hline 28 & $1.50 \pm 0.02$ & $1.60 \pm 0.05$ & $1.75 \pm 0.09$ & $1.80 \pm 0.11$ & $1.55 \pm 0.02$ & $1.69 \pm 0.05$ & $1.80 \pm 0.11$ \\
\hline \multicolumn{8}{|l|}{ ZnONPs } \\
\hline 7 & $1.26 \pm 0.02 \mathrm{~d}$ & $1.42 \pm 0.04 \mathrm{c}$ & $1.54 \pm 0.01 \mathrm{ab}$ & $1.65 \pm 0.08 \mathrm{a}$ & $1.39 \pm 0.01 \mathrm{~cd}$ & $1.48 \pm 0.03 \mathrm{bc}$ & $1.60 \pm 0.05 \mathrm{ab}$ \\
\hline 14 & $1.32 \pm 0.03 \mathrm{~b}$ & $1.49 \pm 0.04 \mathrm{~b}$ & $1.61 \pm 0.05 \mathrm{ab}$ & $1.69 \pm 0.07 \mathrm{a}$ & $1.48 \pm 0.02 \mathrm{bc}$ & $1.58 \pm 0.04 \mathrm{ab}$ & $1.66 \pm 0.04 \mathrm{a}$ \\
\hline 21 & $1.42 \pm 0.01 \mathrm{~b}$ & $1.6 \pm 0.20 \mathrm{ab}$ & $1.71 \pm 0.06 \mathrm{ab}$ & $1.82 \pm 0.06 \mathrm{a}$ & $1.6 \pm 0.01 \mathrm{ab}$ & $1.67 \pm 0.03 \mathrm{ab}$ & $1.79 \pm 0.06 \mathrm{a}$ \\
\hline 28 & $1.50 \pm 0.02 \mathrm{a}$ & $1.75 \pm 0.14 \mathrm{ab}$ & $1.8 \pm 0.09 \mathrm{ab}$ & $1.91 \pm 0.05 \mathrm{a}$ & $1.71 \pm 0.06 \mathrm{ab}$ & $1.78 \pm 0.04 \mathrm{ab}$ & $1.85 \pm 0.08 \mathrm{a}$ \\
\hline
\end{tabular}

Data are presented as mean \pm SE of 6 fish. SE: standard error.

$(\mathrm{a}, \mathrm{b}, \mathrm{c} \ldots$...) means within the same row carrying different letters are significant at $\mathrm{P} \leq 0.05$.

Means having the same letter in the same row are not significantly different.

Table 8. Carbohydrates content (\%) of O. niloticus exposed to $\mathrm{ZnO}$ BPs or $\mathrm{ZnO}$ NPs sub-lethal concentrations and vitamins (E $+\mathrm{C}$ ) supplementation

\begin{tabular}{|c|c|c|c|c|c|c|c|}
\hline \multirow{2}{*}{$\begin{array}{l}\text { Time } \\
\text { (days) }\end{array}$} & \multirow{2}{*}{ Control } & \multicolumn{3}{|c|}{ Sub-lethal concentration } & \multicolumn{3}{|c|}{$\begin{array}{l}\text { Sub-lethal concentration }+ \text { vitamins } \\
\qquad(E+C)\end{array}$} \\
\hline & & $1 / 8 \mathrm{LC}_{50}$ & $1 / 4 \mathrm{LC}_{50}$ & $1 / 2 \mathrm{LC}_{50}$ & $1 / 8 \mathrm{LC}_{50}$ & $1 / 4 \mathrm{LC}_{50}$ & $1 / 2 \mathrm{LC}_{50}$ \\
\hline \multicolumn{8}{|l|}{$\mathrm{ZnOBPs}$} \\
\hline 7 & $0.68 \pm 0.023 \mathrm{a}$ & $0.51 \pm 0.023 \mathrm{bc}$ & $0.59 \pm 0.028 \mathrm{ab}$ & $0.4 \pm 0.057 \mathrm{c}$ & $0.69 \pm 0.051 \mathrm{a}$ & $0.60 \pm 0.086 \mathrm{ab}$ & $0.53 \pm 0.017 \mathrm{abc}$ \\
\hline 14 & $0.56 \pm 0.034 \mathrm{a}$ & $0.31 \pm 0.023 \mathrm{~d}$ & $0.43 \pm 0.028 \mathrm{bc}$ & $0.31 \pm 0.017 \mathrm{~d}$ & $0.53 \pm 0.034 \mathrm{ab}$ & $0.53 \pm 0.040 \mathrm{ab}$ & $0.40 \pm 0.017 \mathrm{c}$ \\
\hline 21 & $0.26 \pm 0.028 \mathrm{ab}$ & $0.28 \pm 0.023 \mathrm{a}$ & $0.19 \pm 0.017 \mathrm{bc}$ & $0.22 \pm 0.023 \mathrm{abc}$ & $0.28 \pm 0.017 \mathrm{a}$ & $0.18 \pm 0.028 \mathrm{c}$ & $0.20 \pm 0.011 \mathrm{bc}$ \\
\hline 28 & $0.34 \pm 0.023 \mathrm{a}$ & $0.11 \pm 0.005 \mathrm{~d}$ & $0.12 \pm 0.011 \mathrm{~d}$ & $0.18 \pm 0.017 \mathrm{~cd}$ & $0.33 \pm 0.028 \mathrm{ab}$ & $0.15 \pm 0.023 \mathrm{~d}$ & $0.25 \pm 0.040 \mathrm{bc}$ \\
\hline \multicolumn{8}{|l|}{$\mathrm{ZnONPs}$} \\
\hline 7 & $0.68 \pm 0.023 \mathrm{a}$ & $0.31 \pm 0.011 \mathrm{c}$ & $0.27 \pm 0.040 \mathrm{de}$ & $0.2 \pm 0.028 \mathrm{e}$ & $0.54 \pm 0.023 \mathrm{a}$ & $0.49 \pm 0.051 \mathrm{bc}$ & $0.43 \pm 0.017 \mathrm{c}$ \\
\hline 14 & $0.56 \pm 0.034 \mathrm{a}$ & $0.33 \pm 0.017 \mathrm{~b}$ & $0.33 \pm 0.017 \mathrm{~b}$ & $0.24 \pm 0.023 \mathrm{~b}$ & $0.55 \pm 0.028 \mathrm{a}$ & $0.50 \pm 0.025 \mathrm{a}$ & $0.51 \pm 0.027 \mathrm{a}$ \\
\hline 21 & $0.26 \pm 0.028 \mathrm{~b}$ & $0.29 \pm 0.023 \mathrm{a}$ & $0.27 \pm 0.011 \mathrm{ab}$ & $0.33 \pm 0.023 \mathrm{ab}$ & $0.34 \pm 0.017 \mathrm{a}$ & $0.30 \pm 0.023 \mathrm{ab}$ & $0.29 \pm 0.011 \mathrm{ab}$ \\
\hline 28 & $0.34 \pm 0.023$ & $0.15 \pm 0.017$ & $0.17 \pm 0.005$ & $0.24 \pm 0.017$ & $0.15 \pm 0.06$ & $0.18 \pm 0.011$ & $0.30 \pm 0.05$ \\
\hline
\end{tabular}

Data are presented as mean \pm SE of 6 fish. SE: standard error.

(a, b, c ...) means within the same row carrying different letters are significant at $\mathrm{P} \leq 0.05$.

Means having the same letter in the same row are not significantly different.

\section{Proximate chemical composition of fish}

Proximate chemical composition in $O$. niloticus muscles exposed to sub-lethal concentrations of $\mathrm{ZnO}$ BPs or $\mathrm{ZnO}$ NPs and supplemented with vitamins $(\mathrm{E}+\mathrm{C})$ after the exposure periods $(7,14,21$, and 28 days) were presented in Tables (4-8). The results showed that moisture contents were insignificantly increased in fish groups exposed to sub-lethal concentrations of $\mathrm{ZnO} \mathrm{BPs}$ or $\mathrm{ZnO}$ NPs compared to control group at all exposure periods. While, a significant increase $(\mathrm{P} \leq 0.05)$ was found in ash content in the fish groups exposed to sub-lethal concentrations of $\mathrm{ZnO} \mathrm{BPs}$ or $\mathrm{ZnO}$ NPs compared to control group at all exposure periods except ash content in fish group exposed to $\mathrm{ZnO}$ BPs at 28 days and fish group exposed to $1 / 8 \mathrm{LC}_{50}$ of $\mathrm{ZnO} \mathrm{NPs}$ at 14 days. Also, ash contents were increased in fish groups exposed to $\mathrm{ZnO}$
NPs more than ZnO BPs at all exposure periods. However, fish exposed to the same concentrations of $\mathrm{ZnO}$ (BPs and NPs) and supplemented with vitamins ( $\mathrm{E}$ and C) have slightly decreased in moisture and ash contents when compared to groups without supplementation at all exposure periods.

On the other hand, protein content revealed a non-significant decrease $(\mathrm{P} \leq 0.05)$ in the fish groups exposed to sub-lethal concentrations of $\mathrm{ZnO}$ NPs at 14 days which decreased significantly $(\mathrm{P} \leq 0.05)$. Also, fish groups exposed to $\mathrm{ZnO}$ (BPs andNPs) have a significant decrease $(\mathrm{P} \leq 0.05)$ in fat content compared to control at all exposure periods except fish group exposed to $\mathrm{ZnO}$ BPs at 14 and 28 days. In addition, protein and fat contents were decreased in fish groups exposed to ZnO NPs more than $\mathrm{ZnO}$ BPs at all exposure periods. On the contrary, 
fish exposed to the same concentrations of $\mathrm{ZnO}$ (BPs andNPs) and supplemented with vitamins ( $\mathrm{E}$ and $\mathrm{C}$ ) have an increase in protein and fat contents when compared to groups without supplementation at the exposure periods. Also, there was a fluctuation in carbohydrates content of all studied fish groups at the exposure periods.

\section{Discussion}

Production and utilization of engineered nanomaterials likely lead to their release into aquatic systems and cause unexpected hazards to aquatic organisms (PeraltaVidea et al., 2011). The effects of nanoparticles on immunity could be dependent on fish species, type, duration, and concentration of nanoparticles (Khoei, 2021). Zinc nanoparticles exist in high levels in the discharges of many industries such as concrete, ceramic and rubber, so this nanometal has a specific interest in fish and the aquatic organisms (Sirelkhatim et al., 2015). Concerns regarding the potential toxic effects of zinc oxide nanoparticles ( $\mathrm{ZnO} \mathrm{NPs}$ ) on aquatic organisms are growing due to the fact that nanoparticles may be released into aquatic ecosystems (Chupani et al., 2018). Zinc metal is taken up by fish directly from water especially by gill and accumulates in the gills of fish and this designates a depressing effect on tissue respiration leading to death by hypoxia. The danger of zinc is serious by its almost unlimited perseverance in the environment because it cannot be destroyed biologically but is only transformed from oxidation state or organic complex to another (Kori-Siakpere and Ubogu, 2008).

In the current study, $\mathrm{Zn}$ accumulation varied with the type of tissue and exposure duration for both sizes where $\mathrm{Zn}$ was concentrated in gill tissue more than muscle tissue. The changes in level of accumulation of different fish tissues are primarily related to the changes in the physiological state of each organ (Karuppasamy, 2004). Muscle tissues accumulated the least zinc levels in this study; muscle tissues contain the skin, which may prevent direct contact with the outside environment. Another reason why muscles are not an active place for metal accumulation may be that muscles do not take part in the detoxification process. This is similar to Uysal et al. (2009) who illustrated that the bioaccumulation level of the muscular tissue was weaker than gill tissue and muscle tissue is considered as an inactive metabolic organ with less accumulating potential.

Accumulation of $\mathrm{Zn}$ in gill tissues may be attributed to the fact that gills serve as respiratory organ by which metal ions are absorbed, stored, and finally transferred to the internal components through blood transport (Bebianno et al., 2004). This agrees with Hao et al. (2013) who reported that zinc accumulated significantly in the exposed gill tissue, which could be a combination of nanoparticles adsorbed directly on the surface of gill with static negative ions and then penetration through gill membrane. Also, it was stated that gills are in direct contact with the aquatic medium so metal levels in this tissue reflect their levels in the external environment and gills might be the target tissues exposed to zinc oxide nanoparticles.

The results of the present study illustrated that fish groups exposed to $\mathrm{ZnO}$ NPs have accumulated higher level of $\mathrm{Zn}$ than that of $\mathrm{ZnO}$ BPs in muscle and gill tissues at all exposure periods. This may indicate that $\mathrm{ZnO}$ NPs could easily penetrate cells with different entering mechanisms. These results are confirmed by Chang et al. (2012) who reported that many routes as ion channels and transporter proteins allow nanoparticles to cross the plasma membrane by endocytosis at which the membrane wraps around nanoparticles and vesicles transport these particles into cells. In addition, Abdel-Khalek et al. (2016) stated that zinc nanoparticles had more efficiency than zinc bulk to penetrate the studied tissues such as the liver, kidneys, gills, skin, and muscle of $O$. niloticus fish. Also, in another study that examined the exposure of carp to nano $(30 \mathrm{~nm})$ and bulk $(2000 \mathrm{~nm})$ forms of $\mathrm{ZnO}$, more $\mathrm{Zn}$ accumulation was also reported for the exposure to nano-size powders (Hao et al., 2013).

Another important finding was that groups of fish exposed to the same sub-lethal concentrations of $\mathrm{ZnO}$ (bulk particles or nanoparticles) and supplemented with vitamins $(E+C)$ have a significant decrease $(P \leq 0.05)$ in accumulated $\mathrm{Zn}$ levels in muscle and gill tissue compared to groups without supplementation. This may be due to the chelating role of vitamins $(\mathrm{E}+\mathrm{C})$ in reducing $\mathrm{Zn}$ content in investigated tissues of $O$. niloticus fish as confirmed by Sahiti et al. (2020) who stated that supplementation of vitamins $\mathrm{C}$ and $\mathrm{E}$ either alone or jointly had significantly decreased $(\mathrm{P}<0.01 ; \mathrm{P}<0.05)$ levels of accumulated heavy metals in investigated tissues of common carp compared to the control and exposed groups.

Moreover, our results illustrated that the values of accumulated $\mathrm{Zn}$ in muscle and gill tissues of supplemented groups returned to similar levels established in the control at low concentrations but still higher than control at the high sub-lethal concentrations of $\mathrm{ZnO} \mathrm{BPs}$ or $\mathrm{ZnO}$ NPs. This indicated that vitamins $(E+C)$ reduced zinc accumulation with low or high concentrations but the greater improvement was observed at low concentrations. Therefore, $\mathrm{Zn}$ accumulation can be reduced by adding vitamins $(\mathrm{E}+\mathrm{C})$. Our results were in agreement with El-Shebly (2009) who stated that treatment of $\mathrm{Pb}$ exposed $O$. niloticus with vitamin E led to a significant decrease in $\mathrm{Pb}$ concentrations in fish tissues. Also, Shahsavani et al. (2017) stated that vitamin C supplementation in Cyprinus carpio exposed to heavy metals reduces the levels of these metals in different tissues. Ebuehi et al. (2012) indicate that oral administration of vitamins $\mathrm{C}$ and E significantly reduced the blood lead concentration. Sahiti et al. (2018) stated that administration of vitamin $\mathrm{C}$ has decreased the concentration of chromium and cadmium but not in significant level and the findings support that dietary vitamin $\mathrm{C}$ supplementation might be 
considered an effective antioxidant against toxic effects of heavy metals in common carp.

Proximate chemical composition evaluation as to moisture content, protein, and lipids is often important to prove that they meet the needs of commercial specifications and food regulations. They also impact on the shelf-life of the fish and post-harvest processing (Jim et al., 2017). The decrease in protein and fat contents of our study is perhaps due to the breakdown of those molecules as energetic substrates to cope with $\mathrm{ZnO}$ BPs or $\mathrm{ZnO}$ NPs which induced stress metabolically and diversification of energy to accomplish impending energy demands as mentioned by Sobha et al. (2007). Moreover, the reduction of lipid and protein contents in fish exposed to $\mathrm{Zn}$ may be due to zinc exposure that induced protein oxidation (Cakmak et al., 2006). In addition, Palaniappan et al. (2010) showed that the exposure to zinc caused important structural changes in the existing proteins indicated by a significant decrease in the $\alpha$-helix intensities. They also stated that the protein secondary structure changed significantly by zinc exposure through reducing the $\alpha$-helix and increasing the $\beta$-sheet content of gills in rohita carp, Labeo rohita.

Increasing of moisture content in the present study may be due to the subsequent utilization of muscle protein and probably an indicator of kidney failure in fish as described by Siddiki et al. (2018). This is similar to results observed by Gaikwad (1981) in Tilapia mossambica exposed to Thiodan and PMA. Also, increasing of ash content may be due to increasing of inorganic residue that likely indicates the accumulation of zinc in fish tissue as mentioned by Siddiki et al. (2018).

Protein and fat contents in the current study were decreased while ash increased in fish groups exposed to $\mathrm{ZnO}$ NPs more than $\mathrm{ZnO}$ BPs at all exposure periods. This could be attributed to the fact that fish groups exposed to $\mathrm{ZnO}$ NPs have higher levels of accumulated $\mathrm{Zn}$ than those exposed to $\mathrm{ZnO}$ BPs as shown in our results which lead to increasing the exposure to Zn. Similarly, Abdel-Tawwab et al. (2013) stated that the contents of whole-body moisture increased significantly, while protein and total lipid contents decreased significantly with increasing $\mathrm{Zn}$ concentrations.

From the results of groups supplemented by vitamins $(E+C)$, there was an improvement in proximate chemical composition tests compared to the other nonsupplemented groups, particularly at the low concentrations. This indicates the ability of both vitamins $(\mathrm{E}+\mathrm{C})$ to overcome the stress induced by $\mathrm{ZnO}$ BPs and $\mathrm{ZnO}$ NPs. This may be due to the fact that vitamins $(\mathrm{E}+\mathrm{C})$ are among the most important nutrients influencing the organism immune system since they will probably protect fish under stress as confirmed by De Andrade et al. (2007). Also, vitamin E has proven beneficial in protecting cellular membrane against oxidation and so increases the resistance to stress (Choi et al., 2004; Farsani et al., 2017). This is similar to Mohamed et al. (2021) who found that vitamins $\mathrm{E}$ and $\mathrm{C}$ have amelio- rative effect and could decrease the toxic effects in O. niloticus.

\section{Conclusion}

It can be concluded from our results that $\mathrm{ZnO}$ NPs showed a high accumulation potency in muscle and gill tissues compared to $\mathrm{ZnO}$ BPs. Zn was accumulated in gill tissue higher than muscle tissue. In addition, fish groups exposed to $\mathrm{ZnO}$ NPs have more affected proximate chemical composition compared to $\mathrm{ZnO}$ BPs. On the other hand, supplementation of vitamins $(E+C)$ decreased levels of accumulated $\mathrm{Zn}$ in investigated tissues compared to groups without supplementation, particularly at the low dose. Also, vitamins $(E+C)$ reduced zinc accumulation and ameliorated chemical composition alterations with low or high concentrations, but greater improvements were observed at low concentrations.

\section{Acknowledgments}

The experimental work was kindly facilitated by Inland Waters and Aquaculture Branch, National Institute of Oceanography and Fisheries (NIOF), Egypt. The authors, therefore, acknowledge with thanks NIOF technical support.

\section{Authors' contributions}

A.S.M. and H.E.G. conceived and designed the experiments; A.S.M. and H.A.S. contributed reagents, materials and analysis tools; A.S.M. and H.E.G. collected data and analysed the data; A.S.M. wrote the manuscript and all other authors contributed to the writing.

\section{Data availability}

All data generated or analyzed during this study are included in this article.

\section{Declarations}

\section{Compliance with ethical standards}

All applicable international, national, and/or institutional guidelines for the care and use of animals were followed.

\section{Competing interests}

The authors declare no competing interests.

\section{References}

Abdelazim A.M., Saadeldin I.M., Swelum A.A.A., Afifi M.M., Alkaladi A. (2018). Oxidative stress in the muscles of the fish Nile tilapia caused by zinc oxide nanoparticles and its modulation by vitamins C and E. Oxid. Med. Cell. Longev., https://doi. org/10.1155/2018/6926712

Abdel-Khalek A.A., Hamed A., Marie M.A. (2016). The accumulation potency of bulk and nano zinc metal and their impacts on the hematological and histological perturbations of Oreochromis niloticus. Water Air Soil Pollut., 227: 206.

Abdel-Tawwab M., Mousaad M.N., Sharafeldin K.M., Ismaiel N.E. (2013). Changes in growth and biochemical status of common carp, Cyprinus carpio L. exposed to water-born zinc toxicity for different periods. Int. Aquat. Res., 5: 11. 
Almeida J.A., Diniz Y.S., Marques S.F.G., Faine L.A., Ribas B.O., Burneiko R.C., Novelli, E.L.B. (2002). The use of the oxidative stress responses as biomarkers in Nile tilapia (Oreochromis niloticus) exposed to in vivo cadmium contamination. Environ. Int., 27: 673-679.

AOAC (2012). Association of Official Analytical Chemists. Official Methods of Analysis. 19th edition, Gaithersburg, Maryland, 20877-2417, USA.

APHA (2005). Standard Methods for the Examination of Water and Wastewater. 21st Edition, American Public Health Association American Water Works Association/Water Environment Federation, Washington DC.

Asaikkutti A., Bhavan P.S., Vimala K., Karthik M., Cheruparambath P. (2016). Effect of different levels dietary vitamin $\mathrm{C}$ on growth performance, muscle composition, antioxidant and enzyme activity of freshwater prawn, Macrobrachium malcolmsonii. Aquac. Rep., 3: 229-236.

Auffan M., Rose J., Bottero J.Y., Lowry G.V., Jolivet J.P., Wiesner M.R. (2009). Towards a definition of inorganic nanoparticles from an environmental, health and safety perspective. Nat. Nanotechnol., 4: 634-641.

Bebianno M.J., Geret F., Hoarau P., Serafim M.A., Coelho M.R., Gnassia-Barelli M., Romeo M. (2004). Biomarkers in Ruditapes decussatus: a potential bioindicator species. Biomarkers, 9: 305-330.

Cakmak G., Togan I., Severcan F. (2006). 17ß-Estradiol induced compositional, structural and functional changes in rainbow trout liver, revealed by FT-IR spectroscopy: a comparative study with nonylphenol. Aquat. Toxicol., 77: 53-63.

Chang Y.N., Zhang M., Xia L., Zhang J., Xing G. (2012). The toxic effects and mechanisms of $\mathrm{CuO}$ and $\mathrm{ZnO}$ nanoparticles. Materials, 5: 2850-2871.

Choi S.W., Benzie I.F.F., Collins A.R., Hannigan B.M., Strain J.J. (2004). Vitamins C and E: acute interactive effects on biomarkers of antioxidant defence and oxidative stress. Mutat. Res., 551: $109-117$.

Chupani L., Niksirat, H., Velíšek J., Stará A., Hradilová Š., Kolařík J., Zusková E. (2018). Chronic dietary toxicity of zinc oxide nanoparticles in common carp (Cyprinus carpio L.): Tissue accumulation and physiological responses. Ecotox. Environ. Safe., 147: 110-116?

Collins D., Luxton T., Kumar, N., Shah S., Walker V.K., Shah V. (2012). Assessing the impact of copper and zinc oxide nanoparticles on soil: a field study. PLoS One., 7, 8:e42663.

De Andrade J.I.A., Ono E.A., de Menezes G.C., Brasil E.M., Roubach R., Urbinati E.C., Tavares-Dias M., Marcon J.L., Affonso E.G. (2007). Influence of diets supplemented with vitamins C and $E$ on pirarucu (Arapaima gigas) blood parameters. Comp. Biochem. Physiol. Part A Mol. Integr. Physiol., 146: 576-580.

Ebuehi O.A.T., Ogedegbe R.A., Ebuehi O.M. (2012). Oral administration of vitamin $\mathrm{C}$ and vitamin $\mathrm{E}$ ameliorates lead-induced hepatotoxicity and oxidative stress in the rat brain. Nig. Q. J. Hosp. Med., 22: 85-90.

El-Sayed A.F.M. (2006). Tilapia Culture. Oceanography Department, Faculty of Science, Alexandria University, Egypt. CABI Publishing, Int. J. Environ. Monit. Annu., 1: 27-33.

El-Shebly A.A. (2009). The role of antioxidant (vitamin E) in the control of lead $(\mathrm{Pb})$ pollution and enhancement of growth within Nile tilapia (Oreochromis niloticus). Int. J. Appl. Res. Vet. M., 7: 97.

Farsani H.G., Doria H.B., Jamali H., Hasanpour S., Mehdipour N., Rashidiyan G. (2017). The protective role of vitamin E on Oreochromis niloticus exposed to ZnONP. Ecotox. Environ. Safe., 145: $1-7$.

Gaikwad S.A. (1981). Toxicity studies with Thiodan 35 EC and Phenyl mercuric acetate on T. mossambica (Peters) Ph. D (Doctoral dissertation, Thesis, Univ. of Bombay).

García-Alonso J., Rodriguez-Sanchez N., Misra S.K., Valsami-Jones E., Croteau M.N., Luoma S.N., Rainbow P.S. (2014). Toxicity and accumulation of silver nanoparticles during development of the marine polychaete Platynereis dumerilii. Sci. Total Environ., 476: 688-695.

Garcia-Santos S., Fontaínhas-Fernandes A., Wilson J.M. (2006). Cadmium tolerance in the Nile tilapia (Oreochromis niloticus) follow- ing acute exposure: assessment of some ionoregulatory parameters. Environ. Toxicol. Int. J., 21: 33-46.

Ghazally K.S. (1988). The bioaccumulation of potential heavy metals in the tissues of the Egyptian edible marine animals. Part 1. Crustaceans [1988], Bull. Natl. Inst. Oceanogr. Fish. (Egypt), 14: 71-77.

Ghazi S., Diab A.M., Khalafalla M.M., Mohamed R.A. (2021). Synergistic effects of selenium and zinc oxide nanoparticles on growth performance, hemato-biochemical profile, immune and oxidative stress responses, and intestinal morphometry of Nile tilapia (Oreochromis niloticus). Biol. Trace Elem. Res., 1-11.]

Hao L., Chen L., Hao J., Zhong N. (2013). Bioaccumulation and subacute toxicity of zinc oxide nanoparticles in juvenile carp (Cyprinus carpio): a comparative study with its bulk counterparts. Ecotox. Environ. Safe., 91: 52-60.

IbrahimD.,Neamat-AllahA.N.,Ibrahim S.M.,EissaH.M.,FawzeyM.M., Mostafa D.I., Abd El-Kader S.A., Khater S.I., Khater S.I. (2021). Dual effect of selenium loaded chitosan nanoparticles on growth, antioxidant, immune related genes expression, transcriptomics modulation of caspase 1, cytochrome $\mathrm{P} 450$ and heat shock protein and Aeromonas hydrophila resistance of Nile tilapia (Oreochromis niloticus). Fish Shellfish Immunol., 110: 91-99.

Jim F., Garamumhango P., Musara C. (2017). Comparative analysis of nutritional value of Oreochromis niloticus (Linnaeus), Nile tilapia, meat from three different ecosystems. J. Food Qual., 2017: 6714347.

Karuppasamy R. (2004). Evaluation of $\mathrm{Hg}$ concentration in the tissue of fish Channa punctatus (Bloch.) in relation to short and longterm exposure to phenyl mercuric acetate. J. Plat. Jubilee AU., 40: 197-204.

Kaya H., Aydin F., Gürkan M., Yilmaz, S., Ates M., Demi,V., Arslan Z. (2015). Effects of zinc oxide nanoparticles on bioaccumulation and oxidative stress in different organs of tilapia (Oreochromis niloticus). Environ. Toxicol. Phar., 40: 936-947.

Kaya H., Aydın F., Gürkan M., Yılmaz S., Ates M., Demir V., Arslan Z. (2016). A comparative toxicity study between small and large size zinc oxide nanoparticles in tilapia (Oreochromis niloticus): Organ pathologies, osmoregulatory responses and immunological parameters. Chemosphere, 144: 571-582.

Khoei A.J. (2021). Evaluation of potential immunotoxic effects of iron oxide nanoparticles (IONPs) on antioxidant capacity, immune responses and tissue bioaccumulation in common carp (Cyprinus carpio). Comp. Biochem. Phys. C., 244: 109005.

Kori-Siakpere O., Ubogu E.O. (2008). Sublethal haematological effects of zinc on the freshwater fish, Heteroclarias sp. (Osteichthyes: Clariidae). Afr. J. Biotechnol., 7: 2068-2073.

Ma H., Williams P.L., Diamond S.A. (2013). Ecotoxicity of manufactured $\mathrm{ZnO}$ nanoparticles - a review. Environ. Pollut., 172: 76-85.

Mahboub H.H., Shahin K., Zaglool A.W., Roushdy E.M., Ahmed S.S.A. (2020). Efficacy of nano zinc oxide dietary supplements on growth performance, immunomodulation and disease resistance of African Catfish, Clarias gariepinus. Dis. Aquat. Org., 142: $147-160$.

Mahboub H.H., Beheiry R.R., Shahin S.E., Behairy A., Khedr M.H.E., Ibrahim S.M., Elshopakey G.E., Daoush W.M., Altohamy D.E., Ismail T.A., El-Houseiny W. (2021). Adsorptivity of mercury on magnetite nano-particles and their influences on growth, economical, hemato-biochemical, histological parameters and bioaccumulation in Nile tilapia (Oreochromis niloticus). Aquat. Toxicol., 235: 105828 .

Mansouri B., Johari S.A., Azadi N.A., Sarkheil M. (2018). Effects of waterborne $\mathrm{ZnO}$ nanoparticles and $\mathrm{Zn} 2+$ ions on the gills of rainbow trout (Oncorhynchus mykiss): bioaccumulation, histopathological and ultrastructural changes. Turk. J. Fish. Aquat. Sci., 18: 739-746?

Mekkawy I.A.A., Mahmoud U.M., Wassif E.T., Naguib M. (2012). Protective roles of tomato paste and vitamin E on cadmium-induced histological and histochemical changes of liver of Oreochromis niloticus (Linnaeus, 1758). J. Fish. Aquat. Sci., 7: 240.

Merrill A., Watt B.K. (1973). Energy value of foods: basis and derivation. Agriculture Handbook, Agricultural Research Service, Washington DC, USA. 
Mohamed A.S., Soliman H.A., Ghannam H.E. (2021). Ameliorative effect of vitamins ( $\mathrm{E}$ and $\mathrm{C}$ ) on biochemical alterations induced by sublethal concentrations of zinc oxide bulk and nanoparticles in Oreochromis niloticus. Comp. Biochem. Phys. C., 108952.

Neamat-Allah A.N.F., Mahmoud E.A., Abd El Hakim Y. (2019). Efficacy of dietary nanoselenium on growth, immune response, antioxidant, transcriptomic profile and resistance of Nile tilapia, Oreochromis niloticus against Streptococcus iniae infection. Fish Shellfish Immunol., 94: 280-287.

Palaniappan P.R., Nishanth T., Renju V.B. (2010). Bioconcentration of zinc and its effect on the biochemical constituents of the gill tissues of Labeo rohita: An FT-IR study. Infrared Phys. Technol., 53, 2: 103-111.

Peralta-Videa J.R., Zhao L., Lopez-Moreno M.L., de la Rosa G., Hong J., Gardea-Torresdey J.L. (2011). Nanomaterials and the environment: a review for the biennium 2008-2010. J. Hazard. Mater. 186: $1-15$.

Rashidian G., Lazado C.C., Mahboub H.H., Mohammadi-Aloucheh R., Proki'c M.D., Nada H.S., Faggio C. (2021). Chemically and green synthesized $\mathrm{ZnO}$ nanoparticles alter key immunological molecules in common carp (Cyprinus carpio) skin mucus. Int. J. Mol. Sci., 22: 3270.

Rundle A., Robertson A.B., Blay A.M., Butler K.M., Callaghan N.I., Dieni C.A., MacCormack T.J. (2016). Cerium oxide nanoparticles exhibit minimal cardiac and cytotoxicity in the freshwater fish Catostomus commersonii. Comp. Biochem. Phys. C., 181: 19-26.

Sahiti H., Bislimi K., Bajgora A., Rexhepi A., Dalo E. (2018). Protective effect of vitamin $\mathrm{C}$ against oxidative stress in common carp (Cyprinus carpio) induced by heavy metals. Int. J. Agric. Biosci., 7: 71-75.

Sahiti H., Bislimi K., Rexhepi A., Dalo E. (2020). Metal accumulation and effect of vitamin $\mathrm{C}$ and $\mathrm{E}$ in accumulated heavy metals in different tissues in common carp (Cyprinus carpio) treated with heavy metals. Pol. J. Environ. Stud., 29: 1.
Sayadi M.H., Pavlaki M.D., Martins R., Mansouri B., Tyler C.R., Kharkan J., Skakari H. (2020). Bioaccumulation and toxicokinetics of zinc oxide nanoparticles ( $\mathrm{ZnO} \mathrm{NPs}$ ) co-exposed with graphene nanosheets (GNs) in the blackfish (Capoeta fusca). Chemosphere, 269: 128689.

Shahsavani D., Baghishani H., Nourian K. (2017). Effect of thiamine and vitamin $\mathrm{C}$ on tissue lead accumulation following experimental lead poisoning in Cyprinus carpio. Iranian J. Vet. Sci. Technol., 9: 39-44.

Siddiki A.N.A., Khair M.U., Naser M.N., Salam M.A. (2018). Biophysicochemical changes in Nile tilapia, Oreochromis niloticus exposed to $\mathrm{ZnSO} 4.7 \mathrm{H} 2 \mathrm{O}$ and $\mathrm{ZnCl} 2$ metal toxicant. J. Innov. Pharm. Biol. Sci.,5: 113-118.

Sirelkhatim A., Mahmud S., Seeni A., Kaus N.H.M., Ann L.C., Bakhori S.K.M., Hasan H., Mohamad D. (2015). Review on zinc oxide nanoparticles: antibacterial activity and toxicity mechanism. Nano Micro Lett., 7: 219-242.

Sobha K., Poornima A., Harini P., Veeraiah K. (2007). A study on biochemical changes in the fresh water fish, Catla catla (Hamilton) exposed to the heavy metal toxicant cadmium chloride. Kathmandu Univ. J. Sci. Eng. Technol., 3: 1-11.

Uysal K., Köse E., Bülbül M., Dönmez M., Erdoğan Y., Koyun M., Ömeroğlu Ç., Özmal F. (2009). The comparison of heavy metal accumulation ratios of some fish species in Enne Dame Lake (Kütahya/Turkey). Environ. Monit. Assess., 157: 355-362.

WHO (2011). The risks and benefits of fish consumption. Report of the joint FAO/WHO expert consultation, 25-29.01.2010, Rome, Italy (No. FIPM/R978 (En)). World Health Organization, p. 50.

Wickham H. (2016). Ggplot2: Elegant graphics for data analysis. New York, NY: Springer-Verlag New York.

Received: 27 IV 2021

Accepted: 15 VI 2021 